\title{
On analogies between algebraic groups and groups of finite Morley rank
}

\author{
Tuna Altmel, Jeffrey Burdges * \\ Université de Lyon \\ Université Lyon 1 \\ CNRS UMR 5208 Institut Camille Jordan \\ 43 blvd du 11 novembre 1918 \\ 69622 Villeurbanne cedex, France
}

November 20, 2018

\begin{abstract}
We prove that in a connected group of finite Morley rank the centralizers of decent tori are connected. We then apply this result to the analysis of minimal connected simple groups of finite Morley rank. Our applications include general covering properties by Borel subgroups, the description of Weyl groups and the analysis of toral automorphisms.
\end{abstract}

\section{Introduction}

Linear algebraic groups and infinite groups of finite Morley rank have many analogous properties. This is expressed in the strongest possible way by the central problem in the analysis of groups of finite Morley rank, namely the Cherlin-Zil'ber algebraicity conjecture which states that an infinite simple group of finite Morley rank is a linear algebraic group over an algebraically closed field. In the last fifteen years an increasing number of partial affirmative answers have been given to special cases of this conjecture. Nevertheless major portions of the problem remain open and counterexamples are not unexpected.

In recent years, results which elucidate strong analogies between algebraic groups and groups of finite Morley rank without proving specific isomorphism theorems have reappeared in the area. Such theorems are reminiscent of the early work of Daniel Lascar and Bruno Poizat, later developed by Frank Wagner. The most important examples are in [17, 24, [4] and [11].

An interesting common point of the main theorems in these four papers is that they permit the introduction of an abstract notion of Weyl group which corresponds in the algebraic category to the usual Weyl group. Several definitions have been suggested and especially 24] and [1] provide keys to the relationships among these possibilites. In this context the centralizers of tori are of importance. The following is known for all connected linear algebraic groups:

Fact 1.1 ([21, Theorem 22.3]) Let $G$ be a connected linear algebraic group and $S$ be a torus in $G$. Then $C_{G}(S)$ is connected.

The thrust of the present work is an analogous result for the centralizers of decent tori in connected groups of finite Morley rank:

Theorem 1 Let $T$ be a decent torus of a connected group $G$ of finite Morley rank. Then $C_{G}(T)$ is connected.

\footnotetext{
* Supported by NSF postdoctoral fellowship DMS-0503036 and a Bourse Chateaubriand postdoctoral fellowship and partially by the ANR project "Groupes, Géométrie et Logique" JC05_47037:jaligot:eric:
} 
Theorem 1 will then be used to obtain several results of varying degrees of generality each of which, in its own way, is in the spirit of the aforementioned recent developments. Our final and more decisive conclusions, denoted theorems, in each of the three final sections of this work depend on minimality conditions which will be explained more in detail in Section 3

In some cases, such minimality conditions are natural because $P S L_{2}$ is the only simple algebraic group where the result holds, as occurs in Section 5 is results on small Weyl groups. There are other cases however where one expects more general results to be true and provable. Our second main result belongs to this latter category:

Theorem 2 Let $G$ be a connected non-solvable group of finite Morley such that if $A$ is a nontrivial definable solvable subgroup of $G$ then $N_{G}^{\circ}(A)$ is solvable. Then every element of $G$ belongs to a Borel subgroup.

The analogous result is known for all connected linear algebraic groups over algebraically closed fields:

Fact 1.2 ([21, Theorem 22.2]) Let $G$ be a connected linear algebraic group and $B$ a Borel subgroup of $G$. Then the union of all conjugates of $B$ is $G$.

In Section 5. we initiate an analysis of Weyl groups in relatively general settings and then draw conclusions on minimal groups. There, minimality is a natural condition under which the structure of the Weyl group is very restricted although still far from being exactly understood. Theorem 3 is the final conclusion of Section 5

Theorem 3 Let $G$ be a minimal connected simple group of finite Morley rank, and let $T$ be a decent torus of $G$. Then the Weyl group $W=N(T) / C(T)$ is metacyclic.

The proof of this theorem involves steps of varying degrees of generality which seem important to us for their own sake. Hence, as the reader of Section 5 will note, we have taken special care to extract the important steps of the main proof as separate conclusions.

Not only do such theorems as Theorem 3 provide a more precise description of general structural properties of minimal groups, but also they are expected to be of use in classification results in the spirit of the Cherlin-Zil'ber conjecture. This is an occurrence of the phenomena in the analysis of groups of finite Morley rank that have been witnessed more frequently since [4, and in that sense the present work is a contribution to the aforementioned line initiated by the work of Poizat and Lascar.

Our final section is devoted to definable groups of automorphisms of groups of finite Morley rank. We obtain general structural information about automorphisms of minimal connected simple groups. For reasons which we detail at the beginning of that section, we feel the conclusions in Section [6 do not only provide specific structural descriptions but also suggest an appraoch to the Cherlin-Zil'ber conjecture in odd type. We prove the following theorem:

Theorem 4 Let $\hat{G}=G A$ be a group of finite Morley rank where $G$ is a definable minimal connected simple subgroup normal in $\hat{G}, A$ is a connected definable abelian subgroup, and $\hat{G}$ is centerless. The following two properties are contradictory.

- $G$ has a divisible abelian torsion subgroup $Q$ with a non-trivial Weyl group $W=N_{G}(Q) / C_{G}(Q)$.

- $\hat{G} / G$ has nontrivial divisible torsion.

On the way to this final theorem, we will prove a more technical statement which suggests an inductive approach to the same result without the minimlity hypothesis. 


\section{Centralizers of tori}

In this section, we prove Theorem 1 The proof necessitates relatively little background beyond basic stable group theory or from the more specialized theory of groups of finite Morley rank. We will use some basic results and notions (types, compactness, eq) from model theory without specific reference. Poizat's books [29] and [30] are excellent sources for model theory in general and the bases of the theory of stable groups. Wagner's book 33] provides technical improvements. As for groups of finite Morley rank, Alexandre Borovik and Ali Nesin's book [6] is our general reference.

The central concept of this section as well as most of the sequel is the notion of a decent torus. To define it, we need the notion of the definable hull of a set in a group of finite Morley rank. For an arbitrary subset $X$ of a group $G$ of finite Morley rank, the definable hull of $X$ with respect to $G$, denoted $d(X)$, is the smallest definable subgroup of $G$ which contains $X$. Its existence is a consequence of the descending chain condition satisfied by the definable subgroups of a group of finite Morley rank. The definable hull of an arbitrary subgroup of a group of finite Morley provides an extension of the notion of connected component. Indeed, if $X$ is an arbitrary subgroup of a group $G$ of finite Morley rank then $X^{\circ}$ is defined as $X \cap d^{\circ}(X)$.

A decent torus in a group of finite Morley rank is a definable, divisible, abelian subgroup which is the definable hull of its torsion. This notion coincides with the usual notion of torus in algebraic groups over algebraically closed fields without additional definable subgroups. In nonzero characteristic, this behavior of multiplicative subgroups of algebraically closed fields holds true in the more general context of fields of finite Morley rank as well:

Fact 2.1 ([34]) Any definable subgroup of the multiplicative group of a field of finite Morley rank of nonzero characteristic is a decent torus.

More generally, any group of finite Morley rank which contains a nontrivial divisible abelian $p$-subgroup (a $p$-torus) for an arbitrary prime $p$ contains a nontrivial decent torus. Indeed, it suffices to consider the definable hull of such a $p$-torus. This strong tie between divisible abelian torsion groups and their definable hulls in groups of finite Morley rank allows one to transfer various results on divisible abelian torsion groups to decent tori. We remind the one about the rigidity of tori which will be used in the sequel without reference:

Fact $2.2([7])$ Let $G$ be a group of finite Morley rank and $T$ be a decent torus in $G$. Then $\left[N_{G}(T): C_{G}(T)\right]<\infty$ and this index is bounded.

This result was proven for $p$-tori and it is immediate for decent tori thanks to the following elementary property of definable hulls in a group $G$ of finite Morley rank: $C(X)=C_{G}(d(X))$ for any $X \subseteq G$.

The following theorem of Cherlin is fundamental:

Fact 2.3 ([12]) Let $G$ be a group of finite Morley rank.

1. If $T$ is a decent torus of $G$ then $\bigcup_{g \in G} C^{\circ}\left(T^{g}\right)$ is generic in $G$.

2. The maximal decent tori in $G$ are conjugate.

A definable subset $X$ of a group of finite Morley rank $G$ is said to be generic in $G$ if finitely many translates of $X$ cover $G$, equivalently if $\operatorname{rk}(X)=\operatorname{rk}(G)$, where rk denotes the Morley rank. A definable subset $X$ of a group of finite Morley rank $G$ is said to be generous if $\bigcup_{g \in G} X^{g}$ is generic in $G$. The following fact is a special case of Lemma 6.2 in [30.

Fact 2.4 ([30, Lemma 6.2]; see also [11, Lemma 1.4]) Let $G$ be a group of finite Morley rank, $A \subseteq G$ a set of parameters, $H$ an $A$-definable normal subgroup of $G$ and $\bar{G}=G / H$. If $a \in G$ is generic over $A$ then its image $\bar{a}$ in $\bar{G}$ is generic over $A$.

By an element generic over a set in a group of finite Morley rank $G$, we mean a realization of a generic type of $G$ over this set of parameters. A type is generic if and only if it contains only generic sets over the set of parameters of the type. 
Before we start the proof of Theorem 1 we also quote two pieces of torsion folklore which have considerable consequences on the behaviour of torsion elements in groups of finite Morley rank.

Fact 2.5 ([6, Ex. 11 p. 93; Ex. 13c p. 72]) Let $G$ be a group of finite Morley rank and let $H \triangleleft G$ be a definable subgroup. If $x \in G$ is an element such that $\bar{x} \in G / H$ is a p-element for some prime $p$, then $x H$ contains a $p$-element. Furthermore, if $H$ and $G / H$ are $p^{\perp}$-groups, then $G$ is a $p^{\perp}$-group.

Fact 2.6 ([6, Ex. 10 p. 93]) Let $G$ be a group of finite Morley rank and $g \in G$. Then $d(g)=$ $A \oplus C$ where $C$ is finite cyclic and $A$ is divisible.

We first prove two lemmas of independent interest. The first one is very much in the spirit of Lemma 1.5 of [11].

Lemma 2.7 Let $G$ be a group of finite Morley rank and take $a \in G$ generic in some coset of $G^{\circ}$. Suppose that $G$ normalizes a maximal decent torus of $C(a)$. Then $d(a)$ contains this decent torus.

Proof. We may assume $G=d(a) G^{\circ}$. Let $T$ be the maximal normal decent torus in $G$. Let $T_{0}$ be the torsion in $T$ and let $R=d(a) \cap T$. As $G^{\circ}$ centralizes $T, R$ does not depend upon $a$, but merely upon $a G^{\circ}$. Thus the definition of $R$ does not necessitate additional parameters to those needed to define $a G^{\circ}$. So, by Fact 2.4 the image $\bar{a}$ of $a$ is generic in $\bar{G}=G / R$. We may therefore assume that $d(a) \cap T=R=1$. So it suffices to show that $S_{0}=C_{T_{0}}(a)$ is finite.

By Fact 2.6 $d(a)=A \oplus C$ is the direct sum of a divisible abelian group $A$ and a finite cyclic group $C$. If $n=|C|$ then for any multiple $N$ of $n$, we have $d\left(a^{N}\right)=A$. On the other hand, for any torsion element $s \in S_{0}$, since $T_{0} \subseteq \operatorname{acl}(\emptyset)$, the element $a^{\prime}=a s$ is also generic over $\emptyset$. Thus, being in the same coset of $G^{\circ}, a^{\prime}$ and $a$ realize the same type. Letting $N$ be a multiple of $n$ and the order of $s$, it follows that $d\left(\left(a^{\prime}\right)^{n}\right)=d\left(\left(a^{\prime}\right)^{N}\right)=d\left(a^{N}\right)=d\left(a^{n}\right)$ and thus $s^{n} \in d\left(a^{n}\right) \leq d(a)$. Now by our reductions $d(a)$ contains no $p$-torus for any $p$, and hence $d(a)$ has bounded exponent. Thus $s^{n}$ has bounded exponent, with $s$ varying and $n$ fixed, and so $S_{0}$ is finite as claimed.

The second preparatory lemma is reminiscent of Fact 2.10 below.

Lemma 2.8 Let $G$ be a group of finite Morley rank. Let $H$ be a definable subgroup of $G$ and $X$ a definable subset of $G$ such that the Morley degree of $X$ is 1 , and

$$
\operatorname{rk}\left(X \cap\left(\bigcup_{g \notin H} X^{g}\right)\right)=\operatorname{rk}(X) .
$$

If $x$ is a realization of the generic type of $X$ then there exists $g \in G \backslash H$ such that $x^{g}$ also realizes the generic type of $X$.

Proof. Let $\Phi(x)$ be the generic type of $X$ over the parameters for $H$ and $X$. We introduce the following set of formulas on the parameters for $H$ and $X$ :

$$
\Psi(x, y)=\{y \notin H\} \cup\left\{X(x), X\left(x^{y^{-1}}\right): X(x) \in \Phi(x)\right\}
$$

Here $X(x)$ ranges over the entire set of formulas in $\Phi(x)$.

We claim that $\Psi(x, y)$ is consistent. Any finite subset of $\Psi(x, y)$ can be assumed to be of the following kind

$$
X_{1}(x) \wedge X_{2}\left(x^{y^{-1}}\right) \wedge y \notin H
$$

where $X_{1}(x)$ and $X_{2}(x)$ are in $\Phi(x)$. Moreover, since $\Phi(x)$ is the generic type of $X$ which is of Morley degree 1 , replacing $X_{1}(x)$ with $X_{1}(x) \wedge X_{2}(x)$ and $X_{2}\left(x^{y^{-1}}\right)$ with $X_{1}\left(x^{y^{-1}}\right) \wedge X_{2}\left(x^{y^{-1}}\right)$ we may assume that we are dealing with $X(x) \wedge X\left(x^{y^{-1}}\right)$ for some $X(x) \in \Phi(x)$. By the generic covering assumption, there exist $b \notin H$ and $a, a_{1} \in X$ such that $a_{1}^{b}=a$. Hence, $(a, b)$ satisfies the formula in question.

By compactness, $\Psi(x, y)$ is realized in an elementary extension of $G$ by $(\alpha, \beta)$. This means that both $\alpha$ and $\alpha^{\beta^{-1}}$ realize $\Phi(x)$. It follows that $\alpha$ is generic in $X$ and $X^{\beta}$. 
In the proof of Theorem 1, we will need several facts on genericity.

Fact 2.9 ([11, Lemma 2.2]) Let $G$ be a group of finite Morley rank, $H$ a connected definable subgroup, and $X$ a definable generic subset of the coset $a H$. Then $\langle X\rangle=\langle a H\rangle=\langle a, H\rangle$.

Fact 2.10 ([4, Lemma 4.1]) Let $G$ be a group of finite Morley rank, $H$ a definable subgroup of $G$, and $X \subseteq G$ a definable set such that

$$
\operatorname{rk}\left(X \backslash \bigcup_{g \notin H} X^{g}\right) \geq \operatorname{rk}(H)
$$

Then $\operatorname{rk}\left(\bigcup X^{G}\right)=\operatorname{rk}(G)$.

Theorem 1 Let $T$ be a decent torus of a connected group $G$ of finite Morley rank. Then $C_{G}(T)$ is connected.

Proof. We may assume that $T$ is a maximal decent torus such that $C(T)>C^{\circ}(T)$. Let $H=C^{\circ}(T)$. There is a $p$-element $w \in C(T) \backslash H$ by Fact 2.5

Consider a realization $a$ of the generic type $\phi(x)$ of $w H$ over the parameter set $T \cup\{w\}$. As $w, H \leq C(T)$, clearly $a \leq C(T)$. For any decent torus $R$ of $C(a)$ containing $T$, we have $a \notin H \geq C^{\circ}(R)$, and $R=T$ by maximality of $T$. So $T$ is a maximal decent torus of $C(a)$. As $T$ is normal in $H, T \leq d(a)$ by Lemma 2.7 .

Before moving ahead, we make a remark about the setwise stabilizer of $w H$, namely the definable subgroup of $G$ :

$$
\operatorname{Stab}(w H)=\left\{g \in G: \operatorname{rk}\left(w H \triangle(w H)^{g}\right)=\operatorname{rk}(w H)\right\} .
$$

By Fact 2.9] $\operatorname{Stab}(w H) \leq N_{G}(\langle w, H\rangle) \leq N_{G}(H)$, the last inclusion being justified by the fact that $H=\langle w, H\rangle^{\circ}$. On the other hand, $N_{G}^{\circ}(H)=C^{\circ}(T)=H \leq \operatorname{Stab}(w H)$. Thus, $\operatorname{Stab}^{\circ}(w H)=H$.

Suppose first that the set $w H \backslash \bigcup_{g \notin \operatorname{Stab}(w H)}(w H)^{g}$ is generic in $w H$. Then, by the remark in the preceding paragraph, $\operatorname{rk}\left(w H \backslash \bigcup_{g \notin \operatorname{Stab}(w H)}(w H)^{g}\right)=\operatorname{rk}(H)=\operatorname{rk}(\operatorname{Stab}(w H))$. By Facts 2.10 and 2.3 (1), a realization $a$ of $\phi(x)$ lies inside $C^{\circ}(S)$ for some maximal decent torus $S$. As $T \leq d(a) \leq C^{\circ}(S)$, we have $T \leq S$. So $a \in C^{\circ}(S) \leq H$, contradicting $w \notin H$. Hence $w H \backslash \bigcup_{g \notin \operatorname{Stab}(w H)}(w H)^{g}$ is not generic in $w H$.

The preceding conclusion implies that $w H \cap\left(\bigcup_{g \notin \operatorname{Stab}(w H)}(w H)^{g}\right)$ is generic in $w H$. For $g \in N_{G}(H) \backslash \operatorname{Stab}(w H)$ then $(w H)^{g} \cap w H=\emptyset$. It follows that the set $w H \cap\left(\bigcup_{g \notin N_{G}(H)}(w H)^{g}\right)$ is generic in $w H$ as well.

At this point, we apply Lemma 2.8 with $X=w H$ and $H$ (in the statement of lemma) for $N_{G}(H)$. This yields $a$ a generic of $w H$, and $b \notin N_{G}(H)$ such that $a^{b}$ is also generic in $w H$. Thus, $T \leq d(a)$ and $T^{b^{-1}} \leq d(a)$ by Lemma 2.7. As $d(a)$ is abelian and $T$ is maximal in $C(a)$, $T^{b}=T$, and $b \in N(T) \leq N_{G}(H)$, a contradiction to the choice of $b$.

\section{Background on minimal groups}

As was mentioned in the introduction, many results proven in the rest of the paper, in particular the final conclusions of the main sections, depend on minimality conditions. The following hypothesis describes the most general minimal setting in which we will be working in the sequel.

Hypothesis 3.1 $G$ is a connected non-solvable group of finite Morley such that if $A$ is a nontrivial definable solvable subgroup of $G$ then $N_{G}^{\circ}(A)$ is solvable.

In literature, one generally encounters a stronger minimality assumption which is the minimal simplicity, namely that $G$ is a connected simple group of finite Morley rank of which the proper 
definable connected subgroups are solvable. In Section [5, we will restrict our analysis to connected minimal simple groups, while in Section 6 the setting described by Hypothesis 3.1 will provide the right context to analyze the automorphisms of minimal connected simple groups.

It is worth noting that Adrien Deloro and Eric Jaligot have undertaken a project which investigates possible generalizations of all results known under Hypothesis 3.1 to a wider setting where the underlying assumption replaces $A$ with $A^{\circ}$. They refer to groups satisfying the weaker hypothesis as locally ${ }^{\circ}$ solvable $^{\circ}$ while the stronger hypothesis referred to by dropping the appropriate $\circ$ sign. We will not use their terminology here to avoid confusion with the traditional use of the phrase "locally solvable".

The main objective of this section is to review some of the results on minimal groups needed in the sequel. Several of these results were proven in the context of minimal simple groups. For the sake of completeness, we will provide their proofs using Hypothesis 3.1 although there are practically no changes. This will be an opportunity to revisit them and occasionally extract additional information from these proofs which is somewhat hidden in their form in print.

The following lemma about the intersections of Borel subgroups is of crucial importance. A Borel subgroup of a group of finite Morley rank is a maximal, definable, connected solvable subgroup. Lemma 3.2 is adapted from [8] where it was proven for minimal connected simple groups.

Lemma 3.2 ([8, Lemma 2.1]) Let $G$ be a group of finite Morley rank satisfying Hypothesis 3.1. Let $B_{1}$ and $B_{2}$ be two distinct Borel subgroups satisfying $U_{p_{i}}\left(B_{i}\right) \neq 1$ where the $p_{i}$ are two prime numbers $(i=1,2)$ which are not necessarily distinct. Then $F\left(B_{1}\right) \cap F\left(B_{2}\right)=1$.

For any group $H$ of finite Morley rank and prime number $p, U_{p}(H)$ denotes its largest, definable, connected, normal, nilpotent $p$-subgroup. In general, definable, connected, nilpotent subgroups of bounded exponent of groups of finite Morley rank are called unipotent subgroups.

We recall two important facts about unipotent $p$-subgroups of groups of finite Morley rank. They will be used in the proof of Lemma 3.2

Fact 3.3 ([26]) Let $H$ be a connected solvable group of finite Morley rank. Then there is a unique maximal unipotent p-subgroup $U_{p}(H)$ of $H$, and $U_{p}(H) \leq F^{\circ}(H)$.

Fact $3.4([27])$ Let $G$ be a nilpotent group of finite Morley rank satisfying $U_{p}(G) \neq 1$. Then $U_{p}(Z(G)) \neq 1$.

Proof of Lemma 3.2. We first show that $U_{p}\left(B_{1} \cap B_{2}\right)=1$ for an arbitrary prime number $p$. Let $X=U_{p}\left(B_{1} \cap B_{2}\right)$ and suppose toward a contradiction that $X \neq 1$. We may assume that $\operatorname{rk}(X)$ is maximal among all choices of $B_{1}$ and $B_{2}$. By Hypothesis 3.1, there is a Borel subgroup $B$ of $G$ containing $N_{G}^{\circ}(X)$.

We will arrive at a contradiction by showing that $B_{i}=B$ for both $i=1,2$. If $X<U_{p}\left(B_{1}\right)$ then Fact 3.3 implies that

$$
X<U_{p}\left(N_{U_{p}\left(B_{1}\right)}^{\circ}(X)\right) \leq U_{p}\left(B \cap B_{1}\right) .
$$

It follows from the maximal choice of $X$ that $B=B_{1}$. Applying the same argument to $B_{2}$ implies that $X=U_{p}\left(B_{2}\right)$ since it has been assumed that $B_{1} \neq B_{2}$. But then, $B_{2} \leq N^{\circ}(X) \leq B \cap B_{2}$ and thus $B=B_{2}$, a contradiction. Thus, $X=U_{p}\left(B_{1}\right)$. A symmetric argument shows that $X=U_{p}\left(B_{2}\right)$. As a result $B_{1}=B=B_{2}$, and this contradiction forces $X=1$.

Having proven that $U_{p}\left(B_{1} \cap B_{2}\right)=1$ for any prime $p$, we proceed to prove the lemma. Suppose toward a contradiction that there is an $f \in F\left(B_{1}\right) \cap F\left(B_{2}\right)$ with $f \neq 1$. Let $Z_{i}=U_{p_{i}}\left(Z\left(F\left(B_{i}\right)\right)\right)$. By Fact 3.4 $Z_{i}$ is a nontrivial unipotent $p_{i}$-subgroup of $Z\left(F\left(B_{i}\right)\right)$ for $i=1,2$. Let $B$ be a Borel subgroup containing $C_{G}^{\circ}(f)$. As $Z_{i} \leq Z\left(F\left(B_{i}\right)\right) \leq C_{G}^{\circ}(f)$ for $i=1,2$, we find $Z_{i} \leq U_{p_{i}}\left(B_{i} \cap B\right)$ for both $i=1,2$. Thus $B_{1}=B=B_{2}$ by the first part.

As a next step in our preparation we adapt a result from [4] to the context of groups satisfying Hypothesis 3.1. This is an occasion for rewording the statement of this fact and extracting some useful information from its proof. 
Fact 3.5 ([4, Proposition 8.1]) Let $G$ be a minimal, connected, simple group of finite Morley rank. Then the set of elements of $G$ which belong to some definable connected nilpotent subgroup of $G$ contains a definable generic subset of $G$.

The proof of Fact 3.5 yields in fact the following proposition:

Proposition 3.6 Let $G$ be a group of finite Morley rank satifying Hypothesis 3.1, Then

- either $G$ does not have torsion,

- or $G$ has a generous Carter subgroup.

Before proving Proposition 3.6, we go over some important notions and facts needed for its proof as well as for later use. A Carter subgroup of a group of finite Morley rank is a definable, connected, nilpotent subgroup of finite index in its normalizer.

\section{Fact 3.7}

1. (17]) Every group of finite Morley rank has a Carter subgroup. The centralizer of a maximal decent torus contains a Carter subgroup of the ambient group.

2. ([32) Carter subgroups of solvable connected groups of finite Morley rank are conjugate.

3. (16]) Carter subgroups of connected solvable groups of finite Morley rank are connected.

4. (16]) Carter subgroups of connected solvable groups of finite Morley rank are self-normalizing.

5. (18]) Carter subgroups of connected solvable groups of finite Morley rank are generous.

For genericity arguments, we will need the following fact in addition to what was recalled in Section 2

Fact 3.8 1. 24, Lemma 2.2] A generous subgroup of a group of finite Morley rank is of finite index in its normalizer.

2. 24, Lemma 2.4] Let $G$ be a group of finite Morley rank and $H$ a connected generous subgroup of $G$. Then any definable generic subset $X$ of $H$ is generous in $G$.

The analysis of torsion elements in the proof of Proposition 3.6 uses the following recent theorem:

Fact 3.9 ([4, Theorem 4], [11]) Let $G$ be a connected group of finite Morley rank, and $a \in G$ a $\pi$-element where $\pi$ is a set of primes. Then $C^{\circ}(a)$ contains an infinite abelian p-subgroup for some $p \in \pi$.

The following lemma is extracted from the proof of Fact 3.5] in [4]:

Lemma 3.10 Let $G$ be a group of finite Morley rank satisfying Hypothesis 3.1, Suppose that $G$ contains no divisible torsion but has nontrivial unipotent torsion. Let $B$ be a Borel subgroup of $G$ with $U_{p}(B) \neq 1$. Then $\bigcup_{g \in G} B^{g}$ is generic in $G$.

Proof. By assumption, $G$ has no nontrivial decent tori but has nontrivial unipotent $p$-subgroups. Then Hypothesis 3.1 and Fact 3.3 imply that $G$ has a Borel subgroup $B$ with $U_{p}(B) \neq 1$. Note that Fact 2.5 and the fact that $G$ does not have decent tori imply that $B / U_{p}(B)$ does not have $p$-torsion. Using Fact 2.1 and Zil'ber's field interpretation results in conjunction with Fact 2.5. one deduces that $B=U_{p}(B) C_{B}\left(U_{p}(B)\right)$.

Next, one proves that for a generic element $g \in B, d(g) \cap U_{p}(B) \neq 1$. Suppose otherwise. Let $z$ be an element of order $p$ in $Z\left(U_{p}(B)\right)$. Then $z g$ is still a generic in $G$. We then have $(z g)^{p}=z^{p} g^{p}=g^{p} \in d(z g) \cap d(g)$. By the assumption on $d(g)$ and Fact 2.5, $d(g) \leq d(z g)$ and it follows that $z \in d(g)$. This contradicts the assumption on $d(g)$.

It follows that $B$ is generically disjoint from its conjugates as otherwise we would have distinct Borel subgroups meeting $U_{p}(B)$ nontrivially, contradicting Lemma 3.2. Since $\left[N_{G}(B): B\right]<\infty$, we can apply Fact 2.10 with $H=N_{G}(B)$ and $X=B$. Thus, $B$ is generous in $G$. 
Now, we proceed to the proof of Proposition 3.6

Proof of Proposition 3.6. We may assume that $G$ has $p$-torsion for some fixed prime $p$. Then by Fact 3.9, $G$ has infinite abelian $p$-subgroups.

We first analyze the case when $G$ has nontrivial decent tori. Let $T$ be a nontrivial decent torus in $G$. By Fact 2.3, $C^{\circ}(T)$ is generous in $G$. By Hypothesis 3.1, $C^{\circ}(T)$ is contained in a Borel subgroup $B$ of $G$. In particular, $B$ is generous in $G$. By Fact 3.7,1, $B$ has Carter subgroups, and by Fact 3.7.5, a Carter subgroup $C$ of $B$ is generous in $B$. By Fact 3.8, $2, C$ is generous in $G$ and it follows from Fact 3.81 , that $\left[N_{G}(C): C\right]<\infty$.

We may assume by the previous paragraph that $G$ has no nontrivial decent tori. As a result of this and Fact 3.9. $G$ has nontrivial unipotent $p$-subgroups. Hence, we can apply Lemma 3.10 to conclude the presence of a generous Borel subgroup $B$. As in the case when $G$ contained nontrivial decent tori, the Carter subgroups of $B$ are generous Carter subgroups of $G$.

\section{Covering by Borel subgroups}

In this section, we prove Theorem 2, As was explained in the introduction, one expects Theorem 2 to hold for all connected groups of finite Morley rank. Here we are content with those satisfying Hypothesis 3.1. The two technical steps of the proof (Lemma 4.3 and Corollary 4.4) seem to be of independent interest.

The following recent theorem is essential for the sequel:

Fact 4.1 ([11, Theorem 3, Corollary 3.3]) Let $G$ be a connected group of finite Morley rank, $\pi$ be a set of primes, a any $\pi$-element of $G$, and suppose $C_{G}^{\circ}(a)$ has $\pi^{\perp}$-type. Then a belongs to any maximal $\pi$-torus of $C(a)$.

Given a set $\pi$ of prime numbers, a group of finite Morley rank is said to be of $\pi^{\perp}$-type if it does not contain nontrivial unipotent $p$-subgroups for any $p \in \pi$.

We also need a well-known fact about the structure of Hall $\pi$-subgroups of connected solvable groups of finite Morley rank:

Fact 4.2 ([6, Theorem 9.29]; [16, Corollaire 7.15]) Hall $\pi$-subgroups of a connected solvable group of finite Morley rank are connected.

One of the main ingredients of the proof is the following lemma which seems valuable in its own right.

Lemma 4.3 Let $G$ be a group of finite Morley rank satisfying Hypothesis 3.1 and $B$ be a Borel subgroup of $G$ such that $U_{p}(B) \neq 1$ for some prime number $p$. Then $p \nmid\left[N_{G}(B): B\right]$.

Proof of Lemma 4.3. Suppose towards a contradiction that $p \mid\left[N_{G}(B): B\right]$ and that this is witnessed by an element $x$ in $N_{G}(B) \backslash B$. Using Fact 2.5 we may assume that $x$ is a $p$ element whose order modulo $B$ is $p$. Let $U$ denote the maximal unipotent $p$-subgroup of $B$. By assumption $U \neq 1$. We will reach a contradiction using the generic behavior of $x B$.

We will apply Fact 2.10 with $X=x B$ and $H=N_{G}(B)$. Let $X_{1}=x B \cap\left(\bigcup_{g \notin N_{G}(B)}(x B)^{g}\right)$. If the set $X_{1}$ is generic in $x B$ then there exists $y \in x B \cap(x B)^{g}$ for some $g \notin N_{G}(B)$. In particular, $y^{p} \in B \cap B^{g}$. Since $x$ normalizes $B$ and $x^{g}$ normalizes $B^{g}, y$ normalizes both $B$ and $B^{g}$. Considering $d(y) \cap B \cap B^{g}$, one can translate $y$ to a $p$-element $z$ by an element of $B \cap B^{g}$ (Fact 2.5). It follows that $z$ centralizes infinite subgroups of both $U$ and $U^{g}$. This forces $g \in N_{G}(B)$ again by Lemma 3.2 This is a contradiction.

If the set $X_{1}$ is not generic in $x B$ then by Fact 2.10 the set $\bigcup(x B)^{G}$ is generic in $G$. At this point we have to consider two possible cases depending on whether or not $G$ has a nontrivial decent torus. 
First, assume that $G$ has a nontrivial decent torus $T$ which we may assume to be maximal. By Fact 2.3, there exist $y \in x B$ and $g \in G$ such that $y \in C^{\circ}\left(T^{g}\right)$ by Fact 2.3. Now we can translate $y$ by an element of $d(y) \cap B$ to a $p$-element $y^{\prime} \in x B$ (Fact 2.5). Since $y^{\prime} \in N_{G}(B)$, $C^{\circ}\left(y^{\prime}\right)$ contains a nontrivial unipotent $p$-subgroup of $B$. Hence, $C^{\circ}\left(y^{\prime}\right)$ is contained in $B$ by Lemma 3.2 .

Since the translation to $y^{\prime}$ is done by an element of $d(y), y^{\prime}$ stays in $C^{\circ}\left(T^{g}\right)$. Let $B_{2}$ be a Borel subgroup of $G$ such that $y^{\prime} \in C^{\circ}\left(T^{g}\right) \leq B_{2}$ (Hypothesis 3.1). Since $y^{\prime}$ is a $p$-element, it is contained in a Sylow $p$-subgroup $P$ of $B_{2}$. Since $B_{2}$ is connected, Fact 4.2 implies that $P$ is connected. If $P$ is a $p$-torus, then $y^{\prime} \in P \leq C^{\circ}\left(y^{\prime}\right) \leq B$. This contradicts that $y^{\prime} \notin B$. Thus $B_{2}$ contains nontrivial unipotent $p$-torsion. Hence, so does $C_{B_{2}}^{\circ}\left(y^{\prime}\right)$. It follows from Lemma 3.2 that $B_{2}=B$, which again forces $y^{\prime} \in B$, a contradiction.

Second, we assume that $G$ has no divisible torsion. Nevertheless, $G$ has $p$-torsion. Thus by Fact 3.9 $G$ has unipotent $p$-torsion. By Lemma 3.10, a Borel subgroup which contains a nontrivial unipotent $p$-subgroup is generous. In our situation $B$ is one such. Hence there exist $y \in x B$ and $g \in G$ such that $y \in B^{g}$. Note that $g \notin N_{G}(B)$ since $y \notin B$. Since $y \in B^{g}$, so is $y^{p}$. Since $y^{p} \in B$ as well, we can repeat the argument which yields a contradiction in the case where $x B$ is generically covered by $\bigcup_{g \notin N_{G}(B)}(x B)^{g}$. No other case remains.

The following corollary is potentially useful:

Corollary 4.4 Let $G$ be a group of finite Morley rank which satisfies Hypothesis 3.1. If $x$ is an element of finite order of $G$ then $x$ lies inside any Borel subgroup $B$ containing $C^{\circ}(x)$.

Proof. By way of contradiction, suppose that $x$ is an element of finite order $m$ for which there exists a Borel subgroup $B$ of $G$ such that $x \notin B$ but $C^{\circ}(x) \leq B$. Choose $x$ so that $m$ is minimal among the orders of all such offending elements. Suppose $m=p_{1}^{\alpha_{1}} \ldots p_{k}^{\alpha_{k}}$ with all the $\alpha_{i}$ different from zero. We set $\pi=\left\{p_{1}, \ldots, p_{k}\right\}$, and for each $i \in\{1, \ldots, k\}$, we let $m_{i}=\frac{m}{p_{i}^{\alpha_{i}}}$ and $x_{i}=x^{m_{i}}$. Note that $x=x_{1} \ldots x_{k}$, and for each $i \in\{1, \ldots, k\},\left|x_{i}\right|=p_{i}^{\alpha_{i}}$.

If $C_{G}^{\circ}(x)$ is of $\pi^{\perp}$-type then it follows from Fact 4.1 that $x \in C_{G}^{\circ}(x)$. Thus, $C_{G}^{\circ}(x)$ contains nontrivial unipotent $p_{i}$-torsion for some $p_{i} \in \pi$. Then by Lemma 3.2 and the standing assumption on $x, x \in N_{G}(B) \backslash B$. Moreover, since $C_{G}(x) \leq C_{G}\left(x_{i}\right)$, it follows from Lemma 3.2 that $C_{G}^{\circ}\left(x_{i}\right) \leq B$. Then, by Lemma 4.3, $x_{i} \in B$. Thus, $|\pi|>1$. As a result, we can apply induction to the other $x_{j}$. Since $C_{G}(x) \leq C_{G}\left(x_{j}\right)$ for every $j \in\{1, \ldots, k\}$, it follows as for $x_{i}$ that $C_{G}^{\circ}\left(x_{j}\right) \leq B$. The minimality of $m$ forces that $x_{j} \in B$. Thus, $x \in B$, a contradiction.

We note that $a$ need not lie inside $C^{\circ}(a)$ if $G$ is a bad group whose Borel subgroups are nilpotent groups constructed by Baudisch ([1]).

Let us restate Theorem 2 before giving its proof which is now immediate.

Theorem 2 Let $G$ be a group of finite Morley rank which satisfies Hypothesis [3.1. Then every element of $G$ belongs to a Borel subgroup.

Proof. Let $x$ be an offending element. By Corollary 4.4 we may assume $x$ is of infinite order. Fact 2.6 yields an $x_{1}$ such that $d(x)=\left\langle x_{1}\right\rangle \oplus d^{\circ}(x)$. By Corollary 4.4, any Borel containing $C_{G}^{\circ}\left(x_{1}\right)$ contains also $x_{1}$. Since $d^{\circ}(x) \leq C_{G}^{\circ}\left(x_{1}\right)$, any Borel containing $x_{1}$ contains $x$ as well.

\section{$5 \quad$ Weyl groups in minimal groups}

We now turn our attention to the smallest Weyl groups. In the context of groups of finite Morley rank, there are several possible ways of defining a Weyl group. Here we will refer to any subgroup of $N_{G}(T) / C_{G}(T)$ where $G$ is a group of finite Morley rank and $T$ is a $\pi$-torus (equivalently, a decent torus) as a Weyl group. It is worth reminding that $C_{G}(T)$ is a connected group by Theorem 1 when $G$ is connected.

Before moving any further, we emphasize that in a connected group $G$ of finite Morley rank if $T$ is a minimal decent torus and $a$ is a representative of a Weyl group element defined with respect 
to $T$ then the torsion subgroup of $C_{T}(a)$ is finite. We shall find below that this condition also holds in minimal connected simple groups of finite Morley rank. So we now turn our attention to its consequences.

Proposition 5.1 Let $G$ be a connected group of finite Morley rank, let $T$ be a $\pi$-torus of $G$, and consider some $W \leq N(T) / C(T)$. Suppose that $C_{T}(a)$ is finite for all $a \in W^{\#}$. Then $W$ is a Frobenius complement.

A Frobenius complement is the stabilizer of a point in a Frobenius group, which is a transitive permutation group such that no non-trivial element fixes more than one point and some nontrivial element fixes a point. Such groups have a quite restrictive structure especially when they are finite (see Fact 5.6 below).

To prove Proposition [5.1, we will need facts from various algebraic sources. The first one is about the Tate module construction of a complex representation.

Fact $5.2([2,[3, \S 3.3])$ Let $T$ be a p-torus of Prüfer p-rank $n$. Then $\operatorname{End}(T)$ can be faithfully represented as the ring $M_{n \times n}\left(\mathbb{Z}_{p}\right)$ of $n \times n$ matrices over the p-adic integers $\mathbb{Z}_{p}$.

The proof of this fact produces an isomorphism $\psi: \operatorname{End}(T) \rightarrow \lim _{k} \operatorname{End}\left(\Omega_{k}(T)\right) \cong M_{n \times n}\left(\mathbb{Z}_{p}\right)$ from the maps $\psi_{k}: \operatorname{End}(T) \rightarrow \operatorname{End}\left(\Omega_{k}(T)\right)$ and the sequence

$$
\operatorname{End}\left(\Omega_{1}(T)\right) \leftarrow \operatorname{End}\left(\Omega_{2}(T)\right) \leftarrow \operatorname{End}\left(\Omega_{3}(T)\right) \leftarrow \cdots
$$

So any $w \in \operatorname{End}(T)$ has an infinite kernel if and only if it has a kernel in each $\operatorname{End}\left(\Omega_{k}(T)\right)$, and then zero as an eigenvalue in $M_{n \times n}\left(\mathbb{Z}_{p}\right)$.

Some well-known results about the representations of finite groups are indispensable for our arguments. Maschke's theorem is one of them:

Fact 5.3 ([19, Corollary 1.6]) Any complex representation of a finite group is a direct sum of irreducible representations.

Another representation theory fact which is crucial for the proof of Proposition 5.1 is Brauer's theorem on splitting fields:

Fact $5.4([\mathbf{2 3}$, Theorem 5.25]) Let $G$ be a finite group of exponent $m$. Then the cyclotomic field $\Lambda^{(m)} / \mathbb{Q}$ of the mth roots of unity is a splitting field for $G$.

For further details we refer the reader to Section 5.12 of 23 .

The proof will involve reduction modulo prime numbers:

Fact 5.5 ([22, Theorem 4.38]) Let $f(x) \in \mathbb{Z}[x]$ be a monic polynomial of degree $n, E$ a splitting field of $f(x)$ over $\mathbb{Q}, p$ a prime such that $f_{p}(x)$ (the image of $f(x)$ after reducing modulo $p$ ) has distinct roots in its splitting field $E_{p}$ over $\mathbb{Z} / p \mathbb{Z}$. Let $D$ be the subring of $E$ generated by the roots of $f(x)$. Then

(a) There exist homomorphisms $\psi$ of $D$ into $E_{p}$.

(b) Any such homomorphism gives a bijection of the set $R$ of roots $f(x)$ in $E$ onto the set $R_{p}$ of roots $f_{p}(x)$ in $E_{p}$.

(c) If $\psi$ and $\psi^{\prime}$ are two such homomorphisms then $\psi^{\prime}=\psi \sigma$ where $\sigma \in \operatorname{Gal}(E / \mathbb{Q})$.

Last but not least before proving Proposition 5.1 is an important notation which will be needed in the sequel as well. For a prime $p$, a $p$-torus, i.e. a divisible abelian $p$-group, is the direct sum of copies of $\mathbb{Z}_{p^{\infty}}$, the group of complex $p^{n}$ th roots of unity under multiplication. When the ambient group is of finite Morley rank, the number of copies is finite for a given $p$-torus $T$ and it is denoted by $p r_{p}(T)$, the Prüfer $p$-rank of $T$.

Proof of Proposition 5.1. Let $T_{p}$ be the maximal $p$-torus in $T$, choosing $p \in \pi$ such that $T_{p} \neq 1$. Since $C_{T}(a)$ is finite for any $a \in W, W \hookrightarrow \operatorname{End}\left(T_{p}\right)$. By Fact 5.2, there is a faithful 
representation of $W$ as $n \times n$ matrices over the $p$-adic integers $\mathbb{Z}_{p}$ where $n=p r_{p}(T)$. By tensoring with $\mathbb{C}$, viewed as the algebraic closure of the fraction field $\mathbb{Q}_{p}$ of $\mathbb{Z}_{p}$, we have a complex representation $V$ of $W$. By Fact [5.3, $V=V_{1} \oplus \cdots \oplus V_{l}$ is a direct sum of irreducible $W$-modules $V_{i}$.

Suppose that some $a \in W^{\#}$ has $C_{V_{i}}(a) \neq 0$. Then 1 is an eigenvalue of $a$. So $1-a \in$ $M_{n \times n}\left(\mathbb{Z}_{p}\right)$ has zero as an eigenvalue. But now $1-a \in \operatorname{End}\left(T_{p}\right)$ has zero as an eigenvalue. So $C_{T_{p}}(a)$ is infinite, a contradiction.

Let $e$ be the exponent of $W$. Since $W$ is a finite subgroup of $\mathrm{GL}_{n}(\mathbb{C}), W$ lies inside $\mathrm{GL}_{n}(\mathbb{Q}[r])$ where $r$ is a primitive $e$ th root of unity (Fact 5.4). We note that $x^{e}-1$ has distinct roots over any field with characteristic relatively prime to $e$ because $x^{e}-1$ is relatively prime to its derivative $e x^{e-1}$. All such roots over $\mathbb{C}$ clearly lie in $\mathbb{Z}[r]$. Let $q$ be a prime coprime to $|W|$. By Fact 5.5 (b), the map $\mathbb{Z}[r] \rightarrow(\mathbb{Z} / q \mathbb{Z})[r]$ induces a bijection on the roots of $x^{e}-1$. For any $w \in W^{\#}$, the characteristic polynomial $p_{w}(x)$ has the form $\Pi_{i=1}^{n}\left(x-\lambda_{i}\right)$ where each eigenvalue $\lambda_{i}$ is a root of $x^{e}-1$. As no $\lambda_{i}$ is 1 in $\mathbb{Z}[r] \leq \mathbb{C}$, their images $\lambda_{i} /(q)$ in $(\mathbb{Z} / q \mathbb{Z})[r]$ under this bijection are also not 1 . In particular $w \neq 1(\bmod q)$ and we still have a faithful representation of $W$ on $V=((\mathbb{Z} / q \mathbb{Z})[r])^{n}$ such that again $C_{V}(a)=0$ for every $a \in W^{\#}$.

Now let $F=V \rtimes W$. Then the group $F$ is a transitive permutation group in its affine action on $V$. We show that $F$ is a Frobenius group with $W$ a Frobenius complement. If some $(b, a) \in F^{\#}$ fixes two points $x, y \in V$ then $a x+b=x$ and $a y+b=y$, and $(a-1)(x-y)=0$. If $x \neq y$ then 1 is an eigenvalue of $a$, so $a=1$ and $b \neq 0$. But when $b \neq 0$, it does not fix any element of $V$. Since $W \cong(0, W) \leq F$ clearly stabilizes $0 \in V, F$ is a Frobenius group. For $f \in F \backslash W,\langle f, W\rangle$ meets $V \cong(V, 1) \leq F$. So $W$ is the whole stabilizer of $0 \in V$, and $W$ is a Frobenius complement.

To apply Proposition 5.1, we need the following basic facts about the structure of a Frobenius complement in finite groups.

Fact $5.6([20,10.3 .1$ p. 339 $])$ Let $W$ be a Frobenius complement in a finite Frobenius group. Then

1. Any subgroup of $W$ of order $p q, p$ and $q$ primes, is cyclic.

2. Sylow p-subgroups of $W$ are either cyclic or possibly generalized quaternion if $p=2$.

3. If $W$ is even, $W$ possesses a unique involution which is central.

As it is clearly visible in the statement of Fact 5.6 the application of Proposition 5.1 together with Fact 5.6 will invoke the Sylow $p$-subgroups of groups of finite Morley rank for various primes $p$. This is a subtle issue, because in its full generality, the Sylow theory in groups of finite Morley rank is well understood only for the case $p=2$. This was achieved by Borovik and Poizat in 7 where the conjugacy of the Sylow 2-subgroups of an arbitrary group of finite Morley rank was proven. Moreover, 7] contained structural information about these subgroups which allowed to divide the groups of finite Morley rank into four mutually disjoint classes: the groups of odd type in which the Sylow 2-subgroups are finite extensions of 2-tori; the groups of even type where the Sylow 2-subgroups are finite extensions of infinite unipotent 2-groups; the groups of mixed type where the Sylow 2-subgroups are finite extensions of a central product of a nontrivial unipotent 2-group and a nontrivial 2-torus; the groups of degenerate type where the Sylow 2-subgroups are finite. An important recent advance in the theory of groups of finite Morley rank is the main theorem of 4] which states that a connected group of finite Morley rank of degenerate type has no involutions. The terminology of types which we have taken a considerable space to review will be used below.

Now, we will investigate the effects of Fact 5.6 in our situation. A finite group is metacyclic if and only if all of its Sylow $p$-subgroups are cyclic [20, 7.6.2 p. 258]. So any Frobenius complement is metacyclic if and only if it does not contain the quaternion group $Q_{8}$ of order 8 , which is clearly contained inside any generalized quaternion group. In particular, connected groups of degenerate type have metacyclic Weyl groups since they contain no involutions by [4]. 
Corollary 5.7 Let $G$ be a connected group of finite Morley rank of degenerate type, $T$ any $\pi$ torus in $G$ and $W=N(T) / C(T)$. If $C_{T}(a)$ is finite for all $a \in W^{\#}$ then $W=N(T) / C(T)$ is metacyclic.

We begin the next part of our analysis with another observation about minimal decent torii, which again clarifies the nature of the problem being discussed. If a minimal decent torus $T$ has a non-cyclic Weyl group, then $\operatorname{pr}_{p}(T) \neq 1$ for any prime $p$ since $W \hookrightarrow \mathbb{Z}_{p}^{*}$ by the above, and $\mathbb{Z}_{p}^{*}$ is cyclic. It follows that such a $T$ is central in any solvable group containing it, by the Zilber Field Theorem.

We will need more detailed information about finite subgroups of algebraic groups over 2-adic numbers. More precisely, we will show that (Proposition 5.9) $\mathrm{GL}_{2}\left(\mathbb{Q}_{2}\right)$ does not have a subgroup isomorphic to the group $Q_{8}$. We imagine that this is well-known to the specialists. Nevertheless, we have not been able to find any written reference for this fact which is provable, as we do below, by elementary means. In general, there is a conceptual and well developed approach to such problems concerning subgroups of algebraic groups over $p$-adic fields (see Proposition 1.12 in 28]) which provides the necessary background to analyze and use the structure of congruence subgroups (see Chapters 5 and 6 in [14]). Unfortunately, in the case of the prime 2, the structure of congruence subgroups is somewhat exceptional which necessitates additional arguments for our question. Thus we have decided to be content with our elementary argument which only needs basic information about 2-adic numbers for which an excellent source is 31 .

Fact 5.8 ([31, Chapitres II et III]) The following are true in $\mathbb{Q}_{2}$ :

1. -1 is not a square.

2. The equation $x^{2}+y^{2}=-1$ does not have a solution.

Proof.

1. This is part of the Corollaire to Théorème 4 in Chapitre II of 31.

2. This uses the Hilbert symbols. Using the notation in Chapitre III of [31, the statement of 2 can be reformulated as $(-1,-1)=-1$ in $\mathbb{Q}_{2}$. Théorème 1 of Chapitre III states that for $(a, b) \in \mathbb{Q}_{2}^{*}$

$$
(a, b)=(-1)^{\epsilon(u) \epsilon(v)+\alpha \omega(v)+\beta \omega(u)}
$$

where $u$ and $v$ are the units in the representation of $a$ and $b$ respectively used also in point 1. The function $\epsilon(u)$ is the residue class of $\frac{u-1}{2}$ modulo 2 . When $a=b=-1$, this statement becomes $(-1,-1)=(-1)^{\epsilon(u)^{2}}$. But $\epsilon(-1)=-1=1$ modulo 2 .

Proposition 5.9 The group $\mathrm{GL}_{2}\left(\mathbb{Q}_{2}\right)$ does not contain an isomorphic copy of the group of quaternions $Q_{8}$.

Proof. Suppose towards a contradiction that $G \leq \mathrm{GL}_{2}\left(\mathbb{Q}_{2}\right)$ is isomorphic to $Q_{8}$. Let $X \in G$ be a matrix of order 4. The minimal polynomial of $X$ is a divisor of $T^{4}-1$ which factorizes as $\left(T^{2}+1\right)(T+1)(T-1)$ over $\mathbb{Q}_{2}$. By Fact 5.81 , these are irreducible factors. As $|X|=4$, it follows that the minimal polynomial of $X$ is $T^{2}+1$. It follows that $X$ is conjugate to $\left(\begin{array}{cc}0 & -1 \\ 1 & 0\end{array}\right)$ in $\mathbb{Q}_{2}$. Therefore, we may assume that $X=\left(\begin{array}{cc}0 & -1 \\ 1 & 0\end{array}\right)$ in $\mathbb{Q}_{2}$.

It follows from the structural properties of $Q_{8}$ that there exists a matrix $\left(\begin{array}{ll}a & b \\ c & d\end{array}\right)$ of order 4 in $\mathrm{GL}_{2}\left(\mathbb{Q}_{2}\right)$ that inverts $X$ by conjugation. By multiplying matrices and using the fact this second matrix is of order 4 , we first conclude that $a=-d$ and $b=c$ and then $a^{2}+b^{2}=-1$. By Lemma 5.8 this equation does not have a solution in $\mathbb{Q}_{2}$, a contradiction. 
We can now constrain the actions upon 2-tori as follows.

Corollary 5.10 Let $G$ be a connected group of finite Morley rank, let $T$ be a $\pi$-torus of $G$, and consider some $W \leq N(T) / C(T)$. Suppose that $C_{T}(a)$ is finite for all $a \in W^{\#}$. If either $\operatorname{pr}_{2}(T)$ is odd or $\operatorname{pr}_{2}(T)=2$ then $W$ is metacyclic.

Proof. By Proposition 5.1, $W$ is a Frobenius complement. Suppose that $W$ is not metacyclic. Then the discussion following Fact [5.6 shows that there is some $W_{0} \cong Q_{8}$ inside $W$. We may also take $p r_{2}(T) \neq 0$ using our hypotheses. Set $n=p r_{2}(T)$ and $V=\mathbb{Q}_{2}^{n}$. As in the proof of Proposition [5.1, there is a faithful representation of $W_{0}$ on $V$ by Fact 5.2, and this becomes a product of irreducible representations over $\mathbb{C}$.

As $Q_{8}$ has 5 conjugacy classes, it has 5 irreducible complex representations: one trivial, three one-dimensional, and the usual two-dimensional one. Only the two-dimensional one satisfies the hypothesis on the centralizers of nontrivial elements of $W$. So our 2-adic representation becomes a product of two-dimensional representations over $\mathbb{C}$, and thus $n$ is even. Our assumption then forces $n=2$, but this is excluded by Proposition 5.9.

We now show that all minimal connected simple groups have metacyclic Weyl groups, which is the culmination of the preceding preparations:

Lemma 5.11 Let $G$ be a connected solvable group of finite Morley rank and $T$ a decent torus. Then $N_{G}(T)$ is connected and $N_{G}(T)=C_{G}(T)$.

Proof. The first statement is a corollary of Facts 3.71, 2 and 3. Indeed, let $x \in N(T)$. Then $x$ normalizes $C^{\circ}(T)$. By Fact $3.71, C^{\circ}(T)$ contains a Carter subgroup $Q$ of $G$. The Frattini argument applied to $C^{\circ}(T)$ and Fact 3.74 yield $N(T)=(N(T) \cap N(Q)) C^{\circ}(T)=Q C^{\circ}(T)=$ $C^{\circ}(T)$.

Theorem 3 Let $G$ be a minimal connected simple group of finite Morley rank, and let $T$ be a decent torus of $G$. Then the Weyl group $W=N(T) / C(T)$ is metacyclic.

In addition to what has been done since the beginning of this section and the recurrent use of Theorem 1, two substantial facts will be invoked in the proof. The first one involves simple groups of odd type:

Fact $5.12([\mathbf{9}, 5$, 10, High Prüfer Rank Theorem $])$ A simple $K^{*}$-group of finite Morley rank with Prüfer 2-rank at least three is algebraic.

A $K^{*}$-group of finite Morley rank is one in which every proper, infinite, definable, simple section is an algebraic group over an algebraically closed field. Note that a minimal counterexample to the Cherlin-Zil'ber conjecture is a $K^{*}$-group. The second fact involves simple groups of even type:

Fact 5.13 ([25]) A simple group of finite Morley rank which contains an infinite elementary abelian 2-subgroup is a linear algebraic group over an algebraically closed field of characteristic 2 .

We have accumulated sufficient arsenal to carry out the proof of Theorem 3, It should be reminded that our key result in this paper, namely Theorem 1 will keep playing its important but quiet role since it is the reason why the centralizers of all decent or $\pi$-tori are connected although this crucial observation is not acknowledged every time it is used.

Proof of Theorem 3. If $G$ has even type, then $G \cong S L_{2}(k)$ where $k$ is an algebraically closed field of characteristic 2 by Fact 5.13 . So we may assume that $G$ is of odd or degenerate type.

The main part of the argument will not distinguish between odd and degenerate types. Let $S$ be a maximal decent torus of $C(T)$, and hence of $G$. By a Frattini argument which uses 
Fact 2.3, $2, N(T)=N_{N(T)}(S) C(T)$. Clearly $C(S) \leq C(T)$ since $T \leq S$. So $W$ is a section of $N(S) / C(S)$. We may thus assume that $T$ is a maximal decent torus of $G$, in particular $\operatorname{pr}_{2}(G)=\operatorname{pr}_{2}(T)$.

For any decent torus $R \leq T, C(R)$ is connected by Theorem 1 and hence solvable. So $N_{C(R)}(T)=C_{C(R)}(T)$ by Lemma 5.11. Thus any $a \in W \cap C(R)$ lies inside $W \cap C(T)$ too, but this second group is trivial. Hence $C_{T}(w)$ has finite torsion for any $w \in W$.

By Corollary [5.7 we are done if $G$ is of degenerate type. Thus, by Corollary [5.10, either $W$ is metacyclic or else $\operatorname{pr}_{2}(T) \geq 4$. But $p r_{2}(G) \leq 2$ by the High Prüfer Rank Theorem, since $G$ is minimal connected simple.

We observe that here one may replace the use of the High Prüfer Rank Theorem, as well as part of the argument, by an application of Adrien Deloro's thesis [13. In a similar vein, one does not need the full force of Fact 5.13 but the analysis of minimal simple connected groups of finite Morley rank, which was nevertheless the most demanding part of the classification of simple groups of even type. We also observe that Theorem 3 should hold under Hypothesis 3.1 assuming that Deloro and Jaligot's program (see the beginning of Section 3) is successful in that context.

\section{Automorphisms of minimal simple groups}

Unlike the High Prüfer Rank Theorem (Fact 5.12), the classification simple groups of finite Morley rank of even type (Fact 5.13 ) is independent of any inductive hypothesis. Its achievement has recently motivated a considerable body of work in the analysis of simple groups of odd type around a similar strategy which consists of carrying out an inductive proof with weaker inductive assumptions. The second author and Cherlin have taken the initiative to move in this direction. The Deloro-Jaligot enterprise mentioned in Section 3 is part of the same picture.

The analysis of simple groups of odd type is more problematic than that of simple groups of even type in that in the latter case the initial step in the induction was rather simple due to the very restricted behavior of unipotent 2 -groups of automorphisms of minimal simple groups. In the case of simple groups of odd type the action of a 2-torus over a minimal connected simple group is far from being understood, and any new technique or tool is worth considering. In this vein, we analize automorphisms of those connected minimal simple groups of finite Morley rank which most resemble algebraic groups. Our viewpoint here is that Theorem 1 forbids outer toral automorphisms in the presence of a Weyl group.

As will be rapidly verified in Lemma 6.2 below, Hypothesis 3.1 offers the right setting for the intended analysis. The following conditions describe the particular properties which lead to Hypothesis 3.1 .

Hypothesis 6.1 Let $\hat{G}=G \rtimes A$ be a group of finite Morley rank where

- $G$ is a definable minimal connected simple subgroup normal in $\hat{G}$,

- A is a connected definable abelian subgroup, and

- $\hat{G}$ is centerless.

Before stating our main theorem, we verify that the group $\hat{G}$ in Hypothesis 6.1 indeed implies Hypothesis 3.1 .

Lemma 6.2 Let $\hat{G}, G$ and $A$ be as in the statement of Hypothesis 6.1. Then $\hat{G}$ satisfies Hypothesis 3.1 .

Proof. Clearly $\hat{G}$ is not solvable. Let $U$ be a nontrivial, definable, abelian subgroup of $\hat{G}$ such that $X=N_{\hat{G}}^{\circ}(U)$ is not solvable. Since $A$ is abelian, $X \cap G \neq 1$ and $X \cap G$ is not solvable. Since $G$ is minimal, either $X \cap G$ is finite or $G \leq X$.

If $X \cap G$ is finite, then since $X^{\prime} \leq X \cap G, X^{\prime}$ is connected and finite. Thus $X^{\prime}$ is trivial which contradicts that $X$ is not solvable.

If $G \leq X$ then $[G, U]=1$. This contradicts that $Z(\hat{G})=1$. 
The following is the main technical step towards the proof of Theorem 4

Proposition 6.3 Let $\hat{G}=G \rtimes A$ be a group of finite Morley rank where both $G$ and $A$ are definable connected subgroups, and $G$ is normal in $\hat{G}$ while $A$ is abelian. Let $Q$ be a definable connected nontrivial subgroup of $C_{G}(A)$. Suppose that $\hat{G} / G$ has nontrivial divisible torsion. Then every coset of $N_{G}(Q) / N_{G}^{\circ}(Q)$ is represented by an element $w$ to which is associated a p-torus $T_{w}$ of $\hat{G}$ such that $\langle w, Q\rangle \leq C_{G}\left(T_{w}\right)$.

Proof. By hypothesis, $\hat{G} / G$ has nontrivial divisible torsion. By Fact 2.5, $A$ has nontrivial divisible torsion which is not contained in $G$.

Since by assumption $[A, Q]=1, C_{\hat{G}}^{\circ}(Q)$ has a nontrivial $p$-torus $T$ for some prime $p$, which we take maximal in $C_{\hat{G}}(Q)$. Furthermore, we may assume that $A=d(A \cap T)$. In particular, $T$ and $A$ commute. So, by a Frattini argument (Fact 2.32) applied to the conjugates of $T$ in $N_{\hat{G}}(Q)$,

$$
N_{\hat{G}}(Q)=N_{N_{\hat{G}}(Q)}(T) C_{\hat{G}}^{\circ}(Q)
$$

On the other hand, since $\hat{G}=G A$ and $A \leq C_{\hat{G}}(Q)$ by assumption, we have

$$
N_{\hat{G}}(Q)=N_{\hat{G}}(Q) \cap G A=N_{G}(Q) A=N_{G}(Q) C_{\hat{G}}^{\circ}(Q) .
$$

It follows from (1) and (2) and the fact that $[A, T]=1$ that for any $x \in N_{\hat{G}}(T) \cap N_{\hat{G}}(Q)$, there exists $y \in G$ such that $x A=y A$ where $y \in N_{G}(Q) \cap N_{\hat{G}}(T)$. Thus $y \in N_{G}(Q) \cap N_{G}(T)$. It follows from this that

$$
N_{N_{\hat{G}}(Q)}(T) C_{\hat{G}}^{\circ}(Q)=N_{N_{G}(Q)}(T) C_{\hat{G}}^{\circ}(Q) .
$$

On the other hand, since we have already shown that

$$
N_{N_{\hat{G}}(Q)}(T) C_{\hat{G}}^{\circ}(Q)=N_{G}(Q) A=N_{G}(Q) C_{\hat{G}}^{\circ}(Q),
$$

we conclude $N_{N_{G}(Q)}(T) C_{G}^{\circ}(Q)=N_{G}(Q)$.

Our initial setup yields $[T G: G]=\infty$. Let $w$ be a representative of a coset of $N_{G}^{\circ}(Q)$ in $N_{G}(Q)$. Since $[w, T] \leq T \cap G<T$, we thus conclude that $C_{T}(w)$ is infinite. We let $T_{w}=C_{T}^{\circ}(w)$ and $H=C_{G}\left(T_{w}\right)$. We have $w \in H$ and $Q \leq C_{G}^{\circ}(T) \leq H$.

We will apply Proposition 6.3 in the minimal context of Hypothesis 6.1 to obtain the following corollary from which the main theorem of this section will immediately follow.

Corollary 6.4 Let $\hat{G}, G$ and $A$ be as in Hypothesis 6.1. Let $Q$ be a definable connected nontrivial subgroup of $C_{G}(A)$ such that for any definable $H \leq G$ containing $Q$, the group $N_{H}^{\circ}(Q)$ is almost self-normalizing. Suppose that $\hat{G} / G$ has nontrivial divisible torsion. Then $N_{G}(Q)$ is connected. In particular, if $Q$ lies inside a Carter subgroup $Q_{1}$ of $G$, then $Q_{1}$ is self-normalizing.

In the proof of Corollary 6.4 we will need a consequence of Frécon's analysis of abnormal subgroups of connected solvable groups of finite Morley rank.

Fact 6.5 ([16, Théorème 1.2]) Let $G$ be a connected solvable group of finite Morley rank and $H$ a definable subgroup of $G$. Then the following are equivalent:

- $H$ is abnormal

- $H$ is definable and connected, and there exist $n \in \mathbb{N}^{*}$ and a decreasing sequence of subgroups $\left(H_{i}\right)_{0 \leq i \leq n}$ such that $H_{0}=G, H_{n}=H$ and for $i>0$ each $H_{i}$ is a proper definable connected maximal subgroup of $H_{i-1}$ not normal in $H_{i-1}$.

A subgroup $H$ of an arbitrary group $G$ is said to be abnormal if $g \in\left\langle H, H^{g}\right\rangle$ for every $g \in G$.

Lemma 6.6 Let $G$ be a connected solvable group of finite Morley rank and $H \leq G$ be a definable connected subgroup of $G$ such that $\left[N_{G}(H): H\right]<\infty$. Then $N_{G}(H)=H$.

Proof. If $H=G$ then there is nothing to do. If $H<G$ then a sequence as in Fact 6.5 can be constructed so that the smallest term is $H$. Thus $H$ is abnormal and hence self-normalizing. 
Proof of Corollary 6.4. The hypotheses of Proposition 6.3 are clearly satisfied. Thus, if $w \in N_{G}(Q) \backslash N_{G}^{\circ}(Q)$, then there exists a $p$-torus $T_{w}$ in $\hat{G}$ which centralizes $w$ and $Q$. By Theorem 1. $C_{\hat{G}}\left(T_{w}\right)$ is connected. Since $\hat{G}=G \rtimes A$, it follows that $H$ is connected. We have $w \in H$ and $Q \leq C_{G}^{\circ}(T) \leq H$. By our hypothesis on $Q, N_{H}^{\circ}(Q)$ is almost self-normalizing in $H$, and hence self-normalizing by Lemma 6.6. So $w \in N_{H}^{\circ}(Q) \leq N_{G}^{\circ}(Q)$, a contradiction.

In particular, taking $Q$ to be a decent torus in a minimal simple group $G$ yields the target theorem of this section:

Theorem 4 Let $\hat{G}, G$ and $A$ be as in Hypothesis 6.1. Then the following two properties are contradictory.

- $G$ has a divisible abelian torsion subgroup $Q$ with a non-trivial Weyl group $W=N_{G}(Q) / C_{G}(Q)$.

- $\hat{G} / G$ has nontrivial divisible torsion.

\section{References}

[1] A. Baudisch. A new uncountably categorical group. Trans. Amer. Math. Soc., 348:3889$3940,1996$.

[2] A. Berkman. The classical involution theorem for groups of finite morley rank. J. Algebra, 243:361-384, 2001.

[3] A. Berkman and A. Borovik. A generic identification theorem for groups of finite Morley rank. J. London Math. Soc., 69 (1):14-26, 2004.

[4] A. Borovik, J. Burdges, and G. Cherlin. Involutions in groups of morley rank of degenerate type. submitted.

[5] A. Borovik, J. Burdges, and A. Nesin. Uniqueness cases in odd type groups of finite Morley rank. J. London Math. Soc. to appear.

[6] A. V. Borovik and A. Nesin. Groups of Finite Morley Rank. Oxford University Press, 1994.

[7] A. V. Borovik and B. Poizat. Tores et p-groupes. J. Symbolic Logic, 55:478-490, 1990.

[8] J. Burdges. The Bender method in groups of finite Morley rank. Submitted.

[9] J. Burdges. Signalizers and balance in groups of finite Morley rank. submitted.

[10] J. Burdges and G. Cherlin a E. Jaligot. Minimal connected simple groups of finite Morley rank with strongly embedded subgroups. J. Algebra. to appear.

[11] J. Burdges and G. Cherlin. Semisimple torsion in groups of finite morley rank. in preparation.

[12] G. Cherlin. Good tori in groups of finite morley rank. J. Group Theory, 8:613-621, 2005.

[13] A. Deloro. Groupes simples connexes minimaux de type impair. PhD thesis, Université Paris 7 - Denis Diderot, 2007.

[14] J. D. Dixon, M. P. F. du Sautoy, A. Mann, and D. Segal. Analytic Pro-p Groups. Cambridge University Press, second edition, 1999.

[15] O. Frécon. Pseudo-tori and subtame groups of finite Morley rank. in preparation.

[16] O. Frécon. Sous-groupes anormaux dans les groupes de rang de Morley fini résolubles. $J$. Algebra, 229:118-152, 2000. 
[17] O. Frécon and E. Jaligot. The existence of Carter subgroups in groups of finite Morley rank. J. Group Theory, 8:623-633, 2006.

[18] O Frécon and E. Jaligot. Conjugacy in groups of finite Morley rank. Cambridge University Press, 2007.

[19] William Fulton and Joe Harris. Representation Theory. Number 129 in Graduate Texts in Mathematics. Springer Verlag, 1991.

[20] D. Gorenstein. Finite Groups. Chelsea Publishing Company, New York, 1980.

[21] J. E. Humphreys. Linear Algebraic Groups. Springer Verlag, Berlin-New York, second edition, 1981.

[22] N. Jacobson. Basic Algebra I. W. H. Freeman and Company, 1989.

[23] N. Jacobson. Basic Algebra II. W. H. Freeman and Company, 1989.

[24] E. Jaligot. Generix never gives up. J. Symbolic Logic, 71:599-610, 2006.

[25] T. Altınel, A. Borovik, and G. Cherlin. Simple Groups of Finite Morley Rank. American Mathematical Society, expected 2007.

[26] A. Nesin. On solvable groups of finite Morley rank. Trans. Amer. Math. Soc., 321:659-690, 1990.

[27] A. Nesin. Poly-separated and $\omega$-stable nilpotent groups. J. Symbolic Logic, 56:694-699, 1991.

[28] Vladimir Platonov and Andrei Rapinchuk. Algebraic Numbers and Number Theory. Academic Press, 1994.

[29] B. Poizat. Cours de Théorie des Modèles. Nur Al-Mantiq Wal-Ma'rifah, Villeurbanne, France, 1985.

[30] B. Poizat. Groupes Stables. Nur Al-Mantiq Wal-Ma'rifah, Villeurbanne, France, 1987.

[31] J.-P. Serre. Cours d'arithmétique. Presses Universitaires de France, Paris, 1970.

[32] F. Wagner. Nilpotent complements and Carter subgroups in stable $\mathfrak{R}$-groups. Arch. Math. Logic, 33:23-34, 1994.

[33] F. Wagner. Stable Groups. Number 240 in London Mathematical Society Lecture Note Series. Cambridge University Press, Cambridge, UK, 1997.

[34] F. Wagner. Fields of finite Morley rank. J. Symbolic Logic, 66:703-706, 2001. 